\title{
Biais artificiels
}

Écrit par : Claire Hathway, L'Observateur de l'OCDE

Dernière mise à jour : 2 mars 2020

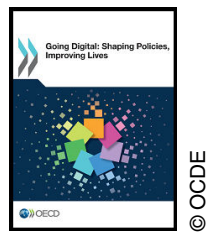

La transformation numérique de la société nous impose d'appliquer nos valeurs à de nouveaux contextes, ce qui ne va pas sans complications. Par exemple, comment assurer l'équité d'un système d'intelligence artificielle (IA) ? Les avantages de l'IA sont clairs : productivité et innovation accrues, données et prédictions plus précises et moins coûteuses. C'est pourquoi des domaines comme la médecine ou la finance y ont déjà recours. Cependant, plus l'IA se généralise, plus ses opérations et ses résultats risquent d'être entachés de biais à caractère sexiste ou raciste.

L'IA, après tout, dépend de l'humain - dans toutes ses contradictions. Les algorithmes d'apprentissage automatique, parce qu'ils interprètent des données qu'on leur a fournies, peuvent émuler les faisceaux de préjugés notamment sexistes et racistes qui s'y trouvent. La persistance de ces biais, en renforçant les dilemmes moraux et éthiques, limite les possibilités actuelles de l'IA qu'on ne pourra continuer de développer que si l'on s'assure qu'elle est impartiale. Mais un système d'IA ne peut être exempt d'un biais que dans la mesure où la personne qui le programme l'est aussi. Il faudra donc déployer des efforts conscients pour surmonter cet obstacle sans perdre les bénéfices des nouvelles technologies.

L'essor des technologies numériques et de traitement des données comme l'IA est le thème de notre nouvelle publication « Going Digital: Shaping Policies, Improving Lives ». Quelles sont les perspectives et les problèmes sous-jacents de la transformation numérique sous sept angles pertinents pour les pouvoirs publics - l'accès, l'utilisation, l'innovation, l'emploi, la prospérité sociale, la confiance, et l'ouverture du marché ? En formulant des recommandations pour chacun, notre publication propose des stratégies de mise en œuvre d'une dématérialisation juste et inclusive.

Il nous semble parfois que les technologies progressent trop vite pour la réglementation. Pourtant, en misant davantage sur la sensibilisation et la 
concertation, les pouvoirs publics, la société civile et les entreprises ont

l'opportunité de modeler le paysage numérique futur et de limiter les effets des biais humains.

\section{Références}

OCDE (2019), Going Digital: Shaping Policies, Improving Lives, Éditions OCDE, Paris, http://dx.doi.org/https://doi.org/10.1787/9789264312012-en. 